\title{
Auger-dominant metastable states in the antiprotonic helium atom.
}

\author{
Vladimir I. Korobov \\ Joint Institute for Nuclear Research \\ 141980, Dubna, Russia
}

\begin{abstract}
Auger dominant metastable states in the antiprotonic helium atoms are studied by using the Complex Coordinate Rotation method. Relativistic corrections for the bound electron related to the Breit interaction have been calculated using CCR wave functions. Higher order relativistic and QED effects have been included into consideration to get precise theoretical values for transition frequencies.
\end{abstract}

Key words: antiprotonic atoms, precise spectroscopy, resonances PACS: 36.10.-k, 31.15.Ar

Metastable states of an exotic atom $\mathrm{He}^{+} \bar{p}$ were of considerable interest in the past years. After first observation at KEK of the delayed annihilation phenomena, when about $3.6 \%$ of antiprotons injected into the helium target [1] survived as long as a few microseconds, precise spectroscopic measurements of several transition lines both in ${ }^{4} \mathrm{He}$ and ${ }^{3} \mathrm{He}$ atoms has been performed at CERN [2,3]. In the recent precise measurements [4] carried out at CERN, in general, the daughter state of the measured transition is the Auger dominant state. That allows to observe a spike in the Annihilation Time Spectra when a laser wavelength is on resonance. To meet the requirements of these experiments it is necessary to perform an accurate study of the Auger dominant states.

Very precise nonrelativistic energies and wave functions have been obtained for the metastable states which decay dominantly via radiative channel [5]. In this case one can effectively apply the Feshbach formalism, when the Hamiltonian is projected onto the subspace of closed channels that still provide with a sufficiently accurate approximation for the wave function. The other advantage is that the standard variational technique may be applied. In case when the Auger decay become dominant the state should be considered as an essentially 
resonant one, and more sophisticated methods are required. We apply the Complex Coordinate Rotation (CCR) method [6] to this problem.

1. Complex Coordinate Rotation. The nonrelativistic Hamiltonian for an antiprotonic helium atom (in atomic units $e=\hbar=m_{e}=1$ ) reads,

$$
H=T+V=-\frac{1}{2 \mu_{\bar{p}}} \boldsymbol{\nabla}_{\mathbf{R}}^{2}-\frac{1}{2 \mu_{e}} \boldsymbol{\nabla}_{\mathbf{r}}^{2}-\frac{1}{m_{\mathrm{He}}} \boldsymbol{\nabla}_{\mathbf{R}} \boldsymbol{\nabla}_{\mathbf{r}}-\frac{2}{R}-\frac{2}{r}+\frac{1}{|\mathbf{R}-\mathbf{r}|},
$$

where $\mathbf{R}, \mathbf{r}$ are the position vectors and $\mu_{\bar{p}}, \mu_{e}$ are the reduced masses of $\bar{p}$ and an electron relative to the helium nucleus, while $T$ and $V$ denote the operators of kinetic and potential energy.

The Coulomb Hamiltonian is analytic under dilatation transformations

$$
(U(\theta) f)(\mathbf{r})=e^{m \theta / 2} f\left(e^{\theta} \mathbf{r}\right), \quad H(\theta)=U(\theta) H U^{-1}(\theta),
$$

for real $\theta$ and can be analytically continued to the complex plane. The ComplexCoordinate Rotation method [6] "rotates" the coordinates of the dynamical system $(\theta=i \varphi), r_{i j} \rightarrow r_{i j} e^{i \varphi}$, where $\varphi$ is the parameter of the complex rotation. Under this transformation the Hamiltonian changes as a function of $\varphi$,

$$
H_{\varphi}=T e^{-2 i \varphi}+V e^{-i \varphi} .
$$

The continuum spectrum of $H_{\varphi}$ is rotated on the complex plane around branch points ("thresholds") to "uncover" resonant poles situated on the unphysical sheet of the Reimann surface in accordance with the Augilar-Balslev-Combes theorem [7]. The resonance energy is then determined by solving the complex eigenvalue problem for the "rotated" Hamiltonian

$$
\left(H_{\varphi}-E\right) \Psi_{\varphi}=0
$$

The eigenfunction $\Psi_{\varphi}$ obtained from Eq. (4), is square-integrable and the corresponding complex eigenvalue $E=E_{r}-i \Gamma / 2$ defines the energy $E_{r}$ and the width of the resonance, $\Gamma$, the latter is being related in case of the antiprotonic helium atoms to the Auger rate as $\lambda_{A}=\Gamma / \hbar$.

It is known that to get an accurate solution for an Auger dominant state in the antiprotonic helium is a pretty difficult task due to a very narrow width of these states and different scales for antiproton and electron orbitals. In our calculations we use a general strategy of a multy-layered variational wave function as it was described in [8]. Details of calculations will be presented elsewhere [9].

2. Leading order relativistic corrections. We consider here only calculation of the spin-independent part of transition energies. The major contribution comes from the relativistic correction for the bound electron,

$$
E_{r c}=\alpha^{2}\left\langle-\frac{\mathbf{p}_{e}^{4}}{8 m_{e}^{3}}+\frac{4 \pi}{8 m_{e}^{2}}\left[Z_{\mathrm{He}} \delta\left(\mathbf{r}_{\mathrm{He}}\right)+Z_{\bar{p}} \delta\left(\mathbf{r}_{\bar{p}}\right)\right]\right\rangle .
$$


The other terms of the Breit Hamiltonian are the relativistic correction to the kinetic energy for heavy particles (with the antiproton Darwin correction),

$$
E_{\text {kin }}=-\alpha^{2}\left\langle\frac{\mathbf{p}_{\mathrm{He}}^{4}}{8 m_{\mathrm{He}}^{3}}+\frac{\mathbf{p}_{\bar{p}}^{4}}{8 m_{\bar{p}}^{3}}-\frac{Z_{\bar{p}}}{8 m_{\bar{p}}^{2}} 4 \pi \delta\left(\mathbf{r}_{\bar{p}}\right)\right\rangle,
$$

the retardation (or the transverse photon exchange) correction,

$$
E_{r e t}=-\alpha^{2} \sum_{i>j} \frac{Z_{i} Z_{j}}{2 m_{i} m_{j}}\left\langle\frac{\mathbf{p}_{i} \mathbf{p}_{j}}{r_{i j}}+\frac{\mathbf{r}_{i j}\left(\mathbf{r}_{i j} \mathbf{p}_{i}\right) \mathbf{p}_{j}}{r_{i j}^{3}}\right\rangle,
$$

and the finite size corrections

$$
E_{\mathrm{FSC}}=\sum \frac{2 \pi Z_{i}\left(R_{i} / a_{0}\right)^{2}}{3}\left\langle\delta\left(\mathbf{r}_{i}\right)\right\rangle,
$$

where $R$ is the root-mean-square radius of the nuclear charge distribution. The RMS radius for the helium nucleus and antiproton is, respectively, $R\left({ }^{4} \mathrm{He}\right)=$ $1.673(1) \mathrm{fm}, R(\bar{p})=0.862(12) \mathrm{fm}$.

The last three contributions are less than the leading contribution from (5) by three or four orders of magnitude. That means that they can be calculated using the Feshbach type closed channel wave function, since a relative accuracy of $\sim 10^{-4}$ is sufficient for these corrections. On contrary, the leading contribution requires more accurate solution, which can be obtained within the framework of the Complex Coordinate Rotation approach.

In order to do that we need a perturbation theory applicable to resonant states. The relevant theory is provided by the theorem proved by Simon [10].

Theorem. Let $H$ be a three-body Hamiltonian with Coulomb pairwise interaction, and $W(\theta)$ be a dilatation analytic perturbation. Let $E_{0}$ be an isolated simple resonance energy (discrete eigenvalue of $H(\theta)$ ). Then for $\beta$ small, there is exactly one resonance of $H+\beta W$ near $E_{0}$ and

$$
E(\beta)=E_{0}+a_{1} \beta+a_{2} \beta^{2}+\ldots
$$

is analytic near $\beta=0$. In particular,

$$
a_{1}=E^{\prime}(0)=\left\langle\Psi_{\theta}^{*}|W(\theta)| \Psi_{\theta}\right\rangle /\left\langle\Psi_{\theta}^{*}, \Psi_{\theta}\right\rangle .
$$

Here a resonance is defined as a complex eigenvalue of $H(\theta)$. In case of the Coulomb three-body system it was proved that these resonances correspond to those which are the "poles" of the $S$-matrix in the scattering theory.

It is obvious that operators encountered in Eq. (5) are dilatation analytic,

$$
\delta_{\theta}(\mathbf{r})=\delta(\mathbf{r}) e^{-3 \theta}, \quad \mathbf{p}_{\theta}^{4}=\mathbf{p}^{4} e^{-4 \theta} .
$$

However they are not "small" perturbations in a sense of the Simon theorem. It is a general practice in QED to regularize these operators in some or other 
way, the only requirement is to preserve a "dilatation analyticity" property. And then after performing all calculations regularization should be removed to get finite results.

\begin{tabular}{lcc}
\hline \hline & $\mathrm{Re}$ & $\mathrm{Im}$ \\
\hline$E_{N R}$ & $-2.847324048(1)$ & $3.709(1) \cdot 10^{-6}$ \\
$\mathbf{p}_{e}^{4}$ & $48.7142(4)$ & $0.0089(4)$ \\
$\delta\left(\mathbf{r}_{\mathrm{He}}\right)$ & $1.60580(1)$ & $0.00029(1)$ \\
$\delta\left(\mathbf{r}_{\bar{p}}\right)$ & 0.05392 & 0.000015 \\
\hline$E_{\text {rel }}$ & $-2.847384180(4)$ & $3.697(4) \cdot 10^{-6}$ \\
\hline \hline
\end{tabular}

Table 1

Relativistic corrections to the energy and Auger width of the $(38,33)$ state of the ${ }^{4} \mathrm{He}^{+} \bar{p}$ atom.

An example of such calculations is shown in Table I, from which is seen that relativistic correction to the Auger decay rate is about $0.3 \%$ and, probably, can be detected in experiment. The final uncertainty of a theoretical value is primarily defined by the uncertainty in the relativistic correction for the bound electon.

3. Higher order corrections and final results. Beyond the corrections described in a previous section there are a few other corrections, which are essential to get reliable theoretical values for transition energies. A list of corrections may be found in [9] and includes QED corrections for the anomalous magnetic moment, self energy and vacuum polarization for the bound electron.

\begin{tabular}{cccc}
\hline \hline transition & this work & experiment [4] & Kino [11] \\
\hline$(33,32) \rightarrow(32,31)$ & $1012445.630(2)$ & $1012445.52(15)$ & $1012445.635(4)$ \\
$(35,33) \rightarrow(34,32)$ & $804633.053(3)$ & $804633.11(10)$ & $804633.09(5)$ \\
$(39,35) \rightarrow(38,34)$ & $501948.765(5)$ & $501949.01(10)$ & $501948.655(7)$ \\
$(36,34) \rightarrow(37,33)$ & $486104.88(3)$ & $486102.7(5)$ & $486104.43(7)$ \\
$(37,34) \rightarrow(38,33)$ & $420120.45(4)$ & $420121.9(7)$ & $420121.53(1)$ \\
$(37,35) \rightarrow(38,34)$ & $412885.129(6)$ & $412885.16(8)$ & $412885.042(7)$ \\
\hline \hline
\end{tabular}

Table 2

Transition frequencies for ${ }^{4} \mathrm{He}^{+} \bar{p}$ atom (in $\mathrm{GHz}$ ) between metastable states in the antiprotonic helium atom for transitions to Auger dominant decay states.

Transition energies for some transitions, which ends up in an Auger-dominant state, are presented in Table 2. The numerical uncertainty in theoretical predictions is finally defined by the numerical uncertainty of the daughter state and further improvement requires significant computational efforts to increase 
substantially an accuracy of the variational wave function for the Augerdominant decay states.

In conclusion we would like to say that while the presented theoretical results are rather accurate still the accuracy is limited mainly by the numerical uncerainty. Thus, for precise spectroscopy study of the three-body QED bound states, it seems more preferable to deal with states and transitions, which lay higher in $(n, l)$ region, and correspond to the states, in which the radiative decay rate exceeds significantly (by some orders of magnitude) the Auger (or resonance) decay rate. Especially that is concerned the two-photon Dopplerfree high precision spectroscopy, which may allow us to determine precisely the antiproton mass, and/or to check the higher order relativistic and QED effects.

Acknowledgements. The author would like to express his gratitude to members of the ASACUSA collaboration for multiple valuable discussions and particularly to R.S. Hayano for support of this contribution to LEAP'03.

\section{References}

[1] M. Iwasaki et al., Phys. Rev. Lett. 67, 1246 (1991).

[2] H.A. Torii et al., Phys. Rev. A, 59, 223 (1999).

[3] T. Yamazaki, N. Morita, R.S. Hayano E. Widmann, and J. Eades, Phys. Reports 366, (2002) 183.

[4] M. Hori et al., Phys. Rev. Lett. 87, 093401 (2001).

[5] V.I. Korobov, D. Bakalov, and H.J. Monkhorst, Phys. Rev. A, 59, R919 (1999).

[6] Y.K. Ho, Phys. Rep. 99, 1 (1983).

[7] J. Aguilar and J.M. Combes, Commun. Math. Phys. 22, 269 (1971); E. Balslev and J.M. Combes, ibid. 22, 280 (1971); B. Simon, ibid. 27, 1 (1972).

[8] V.I. Korobov, Phys. Rev. A, 61, 064503 (2000).

[9] V.I. Korobov, submitted to Phys. Rev. A.

[10] B. Simon, Ann. Math. 97, 247 (1973).

[11] Y. Kino, N. Yamanaka, M. Kamimura, P. Froelich, and H. Kudo, Hyperfine Interact. 138, 179 (2002); and private communications. 